\title{
Successful outcome of pregnancy in a case of aplastic anaemia in Jordan
}

M. Maaita ${ }^{1}$ and A. Telfah ${ }^{2}$

\section{Introduction}

Aplastic anaemia is a rare disease with a major risk of materno-fetal morbidity and mortality [1]. It is characterized by pancytopenia and hypocellular bone marrow [2]. Infections and bleeding remain the main cause of death in these patients [3]. Aplastic anaemia has rarely been described in pregnancy; only a few cases of pregnancy complicated by aplastic anaemia are reported in the literature and most of them had unsuccessful pregnancies $[4,5]$.

Aplastic anaemia may develop during pregnancy and sometimes improve spontaneously after delivery [6]. The etiology is uncertain. However, hormonal imbalance and increased estrogen levels during pregnancy may play a role in the pathogenesis [7]. Alternatively, the pregnancy itself may induce or aggravate bone marrow hypoplasia, which means that the damaged bone marrow of patients with aplastic anaemia cannot respond normally to the stress of pregnancy and the immunosuppression might result in a decrease in circulating blood cells [8].

In most cases of pregnancy-associated aplastic anaemia the only treatment needed is supportive treatment with blood and platelet transfusions as needed and antibiotics when required. A successful outcome can be best accomplished with the close clinical collaboration of the haematologist and the obstetrician. In this paper we report a successful outcome of pregnancy following a diagnosis of aplastic anaemia.

\section{Case report}

A 28-year-old Palestinian woman, parity 2, was referred to the King Hussein Medical Centre from the West Bank of Jordan with the diagnosis of aplastic anaemia at 25 weeks gestation. Her initial presentation was of general weakness, loss of appetite and easy bruising at 16 weeks gestation. There was no history of any medical illness or any drug intake in the past or during pregnancy. Her previous pregnancies were uncomplicated.

On examination, she was found to have pallor. There was no lymphadenopathy or organomegaly. Fundal height of the uterus corresponded with the period of gestation. Ultrasound examination revealed a normal fetus consistent with gestational age. Routine blood count showed haemoglobin of $4.7 \mathrm{~g} / \mathrm{dL}$, white blood cell count $2.1 \times 10^{9} / \mathrm{L}$ and platelets $18 \times 10^{9} / \mathrm{L}$. The reticulocyte count was $0.6 \%$. Serum iron, total iron binding capacity and serum vita$\min B_{12}$ were within normal limits. Viral ti-

${ }^{1}$ Department of Obstetrics and Gynaecology; ${ }^{2}$ Department of Haematology and Oncology, King Hussein Medical Centre, Amman, Jordan.

Received: 14/03/03; accepted: 31/08/03

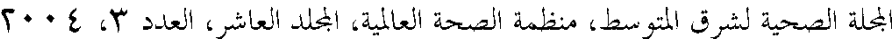


tres were negative to parvovirus, hepatitis A, hepati-tis B and cytomegalovirus. The woman screened negative for human immunodeficiency virus. Bone marrow aspirate and trephine biopsy were markedly hypocellular with decreased numbers of all haemopoietic elements. Myeloid and erythroid maturation was normal. This was consistent with the diagnosis of aplastic anaemia.

The patient was counselled about the severity of the disease and its unpredictable course during pregnancy. However, she decided to continue with the pregnancy. The patient was hospitalized and after 4 units of packed red blood cells transfusion and 4 units of platelets her haemoglobin level increased to $9.5 \mathrm{~g} / \mathrm{dL}$ and platelet count to $55 \times 10^{9} / \mathrm{L}$. Thereafter, although the patient was given $500 \mathrm{mg}$ methyl prednisolone for 3 days intravenously, no apparent benefit was obtained.

The woman was admitted 8 times to hospital throughout the pregnancy. Her main complaint was of general weakness and easy bruising. She was transfused a total of 10 units of packed red blood cells and 40 platelet transfusions to keep her haemoglobin level above $9 \mathrm{~g} / \mathrm{dL}$ and platelet count more than $25 \times 10^{9} / \mathrm{L}$. Despite the high number of admissions, the patient kept reasonably well throughout the pregnancy with no major complications.

The fetus was monitored via routine fetal surveillance tests, which were reassuring until 36 weeks gestation when the baby was showing signs of growth restriction. Fetal Doppler showed normal cardiac signs.

Dexamethazone was given and birth was induced at 38 weeks of gestation with intravaginal prostaglandin $\mathrm{E}_{2}$ gel. Steroids and prophylactic antibiotics were started and single donor platelet transfusion was made at the time of induction. The patient also received 3 units of blood. Her haemoglobin level was $9.5 \mathrm{~g} / \mathrm{L}$ and platelet count was $63 \times 10^{9} / \mathrm{L}$. After 8 hours of labour, she delivered vaginally a healthy male baby weighing $2050 \mathrm{~g}$ with good Apgar scores. She did not have any postpartum haemorrhage or infection. She was started on cyclosporin $300 \mathrm{mg} /$ day and was discharged home on the 4th day after delivery.

At 1 month follow-up, the patient's haemoglobin was $9.5 \mathrm{~g} / \mathrm{dL}$, white blood cell count $5.1 \times 10^{9} / \mathrm{L}$ and platelet count $65 \times 10^{9} / \mathrm{L}$. Following this, she returned to the West Bank area and was lost to followup.

\section{Discussion}

Aplastic anaemia has rarely been described in pregnancy. It is a very serious event threatening the lives of both the mother and the fetus. The mortality is more than $20 \%$ and is mainly due to haemorrhage and sepsis [9]. The etiology is uncertain, and the treatment of choice, bone marrow transplant, is contraindicated in pregnancy. Thus, the occurrence of this complication during gestation presents a management challenge [7].

Pre-existing aplastic anaemia associated with pregnancy has a better prognosis than when it occurs during pregnancy [10]. The relationship between pregnancy and aplastic anaemia has become a matter of speculation. There is no conclusive evidence to implicate pregnancy as an etiological factor for aplastic anaemia [11]. However, some researchers have supported the association of pregnancy with aplastic anaemia by the fact that it can relapse in subsequent pregnancies and it resolves with pregnancy termination $[12,13]$. Because of the medications required, fetal haemorrhage and infection risks especially during the pregnancy, the patient with a plastic anaemia should

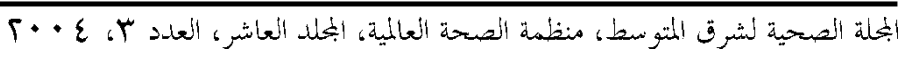


be counselled about the dangers of pregnancy. If the disease is detected in the first trimester, pregnancy termination should be considered [9].

The management of aplastic anaemia in pregnancy includes the treatment of the disease itself with supportive management, which will depend on the severity of the aplastic anaemia and consideration for the welfare of the fetus. Clearly, the stage of the pregnancy and the patient's wishes must be taken into account. Bone marrow transplant is definitely contraindicated in pregnancy because the pre-transplant immunosuppressive regime is toxic to the developing fetus. Bone marrow transplant must therefore be delayed until the pregnancy is terminated or until after delivery [13]. Continuous intensive haematological support given repeatedly during pregnancy remains the best management for these patients. White blood cell transfusion is required only in severe infections. Ideally, HLA-matched platelets or single donor platelet transfusion should be transfused to prevent cross-immunization [14]. Steroids, antithymocyte globulin therapy and cyclosporin are the other treatments available to resolve haematopoiesis [15].

Our patient had a successful outcome but was unfortunately lost to follow-up. She was warned about the possible need for bone marrow transplant in the future.

In conclusion, intensive haematological support given repeatedly during the pregnancy and close liaison between haematologist and obstetrician was of prime importance in the successful management of this case.

\section{References}

1. Ohba T et al. Aplastic anaemia in pregnancy: treatment with cyclosporine and granulocyte-colony stimulating factor. Acta obstetricia et gynecologica scandinavica, 1999, 78:458-61.

2. Young NS, Maciejewski J. The pathophysiology of acquired aplastic anaemia. New England journal of medicine, 1997, 336:1365-72.

3. Williams DM. Pancytopenia, aplastic anaemia and pure red cell aplasia. In: Lee GR et al., eds. Wintrobe's clinical haematology. Philadelphia, Williams and Wilkins, 1999:1449.

4. Van Besien K et al. Pregnancy-associated aplastic anemia-report of 3 cases. European journal of haematology, 1991, 47(4):253-6.

5. Leong KW. Successful pregnancy following aplastic anaemia. Postgraduate medical journal, 1995, 71:625-7.
6. Cunningham FG et al. Williams obstetrics, 20th ed. Norwalk, Connecticut, Appleton and Lange, 1997.

7. Fleming AF. Hypoplastic anaemia in pregnancy. British medical journal, 1973, 3:166-7.

8. Pajor A et al. Pregnancy in idiopathic aplastic anaemia (report of 10 patients). European journal of obstetrics, gynecology, and reproductive biology, 1992, 45:19-25.

9. Aitchison RG et al. Pregnancy associated with aplastic anaemia: a report of five cases and review of current management. British journal of haematology, 1989, 73:541-5.

10. Pavithran K, Thomas M. Pregnancy associated aplastic anaemia. Journal of the Association of Physicians of India, 1996, 44:273.

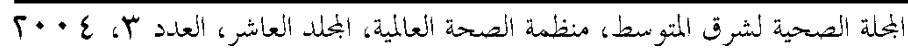


11. Oosterkamp HM et al. Pregnancy and severe aplastic anaemia: causal relation or coincidence? British journal of haematology, 1998, 103:315-6.

12. Goldstein IM, Coller BS. Aplastic anaemia in pregnancy: recovery after normal spontaneous delivery. Annals of internal medicine, 1975, 82:537-9.

13. Bourantas $\mathrm{K}$ et al. Aplastic anaemia: a report of a case with recurrent episodes in consecutive pregnancies. Journal of reproductive medicine, 1997, 42:672-4.
14. Ascarelli $\mathrm{MH}$ et al. Aplastic anaemia and immune-mediated thrombocytopenia: concurrent complications encountered in the third trimester of pregnancy. $O b$ stetrics and gynecology, 1998, 91:8036.

15. Deka D et al. Aplastic anaemia during pregnancy: variable course and outcome. European journal of obstetrics, gynecology, and reproductive biology, 2001, 94:152-4.

\section{Managing complications in pregnancy and childbirth. A guide for midwives and doctors now available in Arabic}

Managing complications in pregnancy and childbirth. A guide for midwives and doctors is now available in Arabic. This manual is written for midwives and doctors at the district hospital who are responsible for the care of women with complications of pregnancy, childbirth or the immediate postpartum period including immediate problems of the newborn. The emphasis of the manual is on rapid assessment and decision making. The clinical action steps are based on clinical assessment with limited reliance on laboratory or other tests and most are possible in a variety of clinical settings (e.g. district hospital or health centre). The Arabic translation is available from: World Health Organization Regional Office for the Eastern Mediterranean, Abdul Razzak Al Sanhouri Street, P.O. Box 7608, Masr City, Cairo 11371, Egypt (Telephone: (202) 67025 35; Facsimile: (202) 6702492 or 6702494 ). 